\title{
ON GROUPS OF EXPONENT FOUR. II
}

\author{
C. K. GUPTA AND N. D. GUPTA
}

\begin{abstract}
C. R. B. Wright has shown that the nilpotency class of an $n$-generator group of exponent four is at most $3 n-1$. This bound is believed to be too large for higher values of $n$. In this note it is shown that if for some large enough integer $n$ this bound can be improved to $\left[2 \frac{1}{2} n\right]-1$, then the free group of exponent four of infinite rank is solvable.
\end{abstract}

Introduction. Let $\kappa(n)$ denote the nilpotency class of $F_{n}\left(\mathfrak{B}_{4}\right)$. Wright [3] has shown that $\kappa(n) \leqq 3 n-1$. For higher values of $n$ this bound is believed to be too large. The purpose of this note is to point out a connection with the solvability problem of $F_{\infty}\left(\mathfrak{B}_{4}\right)$ studied in Gupta-Weston [2]. Our main result may be stated as follows:

THEOREM. Let $N$ be a fixed positive integer such that for all $n \geqq N$, $\kappa(n) \leqq\left[2 \frac{1}{2} n\right]-1$, then $F_{\infty}\left(\mathfrak{B}_{4}\right)$ is solvable of bounded length.

Notation. We use standard notation: $[x, y]=x^{-1} y^{-1} x y ;[x, y, z]=$ $[[x, y], z] ;[x, y ; u, v]=[[x, y],[u, v]] ; G^{4}$ is the subgroup of $G$ generated by 4th powers of elements of $G ; W(G)$ is the verbal closure of a word $w$ in $G ; \gamma_{n}(G)$ is the $n$th term of the lower central series of $G ; \delta_{m}(G)$ is the $m$ th term of the derived series of $G$; $\left\langle x^{G}\right\rangle$ is the normal closure of $x$ in $G$; $\mathfrak{B}_{4}$ is the variety of groups of exponent $4 ; F_{n}\left(\mathfrak{B}_{4}\right)$ is the free $\mathfrak{B}_{4}$-group of rank $n ; F=F_{\infty}$ is the free group of infinite rank.

Preliminaries. Any $r$ variable commutator word of weight $s$ is said to be of type $(r \rightarrow s)$. Consider

$$
w_{0}=\left[z_{0}, y_{0}, z_{0}, x_{0}, x_{0}, y_{0}, y_{0}\right]
$$

to be a word of type $(3 \rightarrow 7)$. Using $w_{0}$ as a base we shall define words $w_{n}$ of type $\left(2 \cdot 2^{n+1}-1 \rightarrow 5 \cdot 2^{n+1}-3\right)$ inductively. Let $k \geqq 0$ and assume we have already defined $w_{k}$ of type $\left(2 \cdot 2^{k+1}-1 \rightarrow 5 \cdot 2^{k+1}-3\right)$. Let $w_{k 1}, w_{k 2}$ be two copies of $w_{k}$ involving nonoverlapping variables; and define

$$
w_{k+1}=\left[w_{k 1}, y_{k+1}, w_{k 2}, y_{k+1}, y_{k+1}\right]
$$

which is clearly of type $\left(2 \cdot 2^{k+2}-1 \rightarrow 5 \cdot 2^{k+2}-3\right)$.

Received by the editors January 5, 1971.

AMS 1969 subject classifications. Primary 2008, 2040.

Key words and phrases. Exponent four, nilpotency class, solvability, commutator, verbal closure, free group, variety.

(c) American Mathematical Society 1972 
For our later use we record the following:

Lemma 1. Modulo $F^{4}$, (i) $\left\langle w_{n}^{F}\right\rangle$ is abelian; (ii) $w_{n}^{2}=1$ for all $n \geqq 0$.

Proof of (i). The derived group of $\left\langle w_{n}^{F}\right\rangle$ is the normal closure of all elements of the form $\left[w_{n}, x, w_{n}\right]$. Since the weight of this commutator is more than three times the number of variables involved, by the result of Wright $[3],\left[w_{n}, x, w_{n}\right]=1$.

Proof OF (ii). By the Corollary on p. 220 of Gupta-Tobin [1], $w_{0}^{2}$ is a product of commutators of weight at least 9 in 3 variables, each of which is trivial by the result of Wright. Thus $w_{0}^{2}=1$ and together with (i), $\left\langle w_{0}^{F}\right\rangle$ is of exponent 2. Since, for each $n \geqq 0, w_{n} \in\left\langle w_{0}^{F}(n)\right\rangle$ for some copy $w_{0}(n)$ of $w_{0}$, it follows that $w_{n}^{2}=1$. This completes the proof of Lemma 1 .

Let $H$ be a group of exponent 4 generated by $x_{1}, x_{2}, \cdots$ and satisfying only the following relations and their consequences:

(I) $x_{i}^{2}=1$,

(II) $\left[x_{i}, h, x_{i}\right]=1$ for all $i=1,2, \cdots$ and all $h \in H$.

LeMma 2 (Gupta-Weston [2]). (i) $H$ satisfies $\left[x_{i}, h, h, h\right]=1$ for all $i=1,2, \cdots$ and all $h \in H$.

(ii) $\delta_{k}(H)=\{1\}$ implies $\delta_{k+2}(F)<F^{4}$.

(iii) If $H$ satisfies the relations $\left[x_{i}, h, x_{j}, h, h\right]$ for all $i, j \in\{1,2, \cdots\}$ and all $h \in H$, then $H$ is centre-by-metabelian. ((iii) follows from the proof of Theorem 2 of [2].)

Lemma $3 .^{1}$ Let $h$ be an arbitrary but fixed element of $H$ and let $K_{n}$ $(n \geqq 1)$ denote the subgroup of $H$ generated by $x_{1}, \cdots, x_{n}, h$. Then $\gamma_{n+4}\left(K_{n}\right)=\{1\}$.

Proof. We may assume that $\gamma_{n+5}\left(K_{n}\right)=\{1\}$. Since $\left\langle x_{i}^{H}\right\rangle$ is abelian for each $i, \gamma_{n+4}\left(K_{n}\right)$ is generated by commutators of weight $n+4$ each of which contains at least four $h$ 's. Thus for $n \geqq 2, \gamma_{n+4}\left(K_{n}\right)=\{1\}$ by Theorem 1 of Wright [3] and for $n=1, \gamma_{5}\left(K_{1}\right)=\{1\}$ by Lemma 2(i).

Lemma 4. $\delta_{4}(H) \leqq W_{0}(H)$.

Proof. Assume $W_{0}(H)=\{1\}$, so that $H$ satisfies $w_{0}=1$. In particular for all $h \in H$,

$$
\begin{aligned}
1 & =\left[x_{1} x_{2}, h, x_{1} x_{2}, x_{3} x_{4}, x_{3} x_{4}, h, h\right] \\
& =\left[x_{1}, h, x_{2}, x_{3} x_{4}, x_{3} x_{4}, h, h\right]\left[x_{2}, h, x_{1}, x_{3} x_{4}, x_{3} x_{4}, h, h\right] \\
& \left.=\left[\left[x_{1}, x_{2}\right], h, x_{3} x_{4}, x_{3} x_{4}, h, h\right] \quad \text { (by Jacobi identity and Lemma } 3\right) \\
& =\left[\left[x_{1}, x_{2}\right], h, x_{3}, x_{4}, h, h\right]\left[\left[x_{1}, x_{2}\right], h, x_{4}, x_{3}, h, h\right] \\
& =\left[\left[x_{1}, x_{2}\right], h,\left[x_{3}, x_{4}\right], h, h\right] \quad \text { (by Jacobi identity and Lemma 3). }
\end{aligned}
$$

${ }^{1}$ The authors wish to thank the referee for suggesting Lemma 3 which has simplified the proof of Lemma 4. 
By Lemma 2(iii), $\delta_{1}(H)$ is centre-by-metabelian and in particular $\delta_{4}(H) \leqq W_{0}(H)$.

LEMMA 5 . $\delta_{3 n+4}(H) \leqq W_{n}(H)$ for all $n \geqq 0$.

Proof. By induction on $n$. For $n=0$, the result comes from Lemma 4 . For the inductive step we assume $\delta_{3 k+4}(H) \leqq W_{k}(H)$ and proceed to conclude that $\delta_{3 k+7}(H) \leqq W_{k+1}(H)$. We may assume $W_{k+1}(H)=\{1\}$ so that $H$ satisfies the law $w_{k+1}=\left[w_{k 1}, h, w_{k 2}, h, h\right]$. Let $\bar{H}$ denote the subgroup of $H$ generated by $w_{k 1}, w_{k 2}, \cdots, w_{k 5}$ where each $w_{k i}$ is a copy of $w_{k}$. By Lemma $1, \bar{H}$ is a homomorphic image of $H$ and, by Lemma $2, \bar{H}$ is centreby-metabelian. Thus $\delta_{3}\left(W_{k}(H)\right) \leqq W_{k+1}(H)$ and by induction hypothesis $\delta_{3 k+7}(H)=\delta_{3}\left(\delta_{3 k+4}(H)\right) \leqq \delta_{3}\left(W_{k}(H)\right) \leqq W_{k+1}(H)$. This concludes the proof of Lemma 5.

Proof of The Theorem. Choose $n \geqq N$ of the form $2 \cdot 2^{l+1}-1$. Since $\kappa(n) \leqq\left[2 \frac{1}{2} n\right]-1$, every commutator of weight [2 $\left.\frac{1}{2} n\right]$ in $n$ variables is trivial $\bmod F^{4}$. In particular every commutator of type $\left(2 \cdot 2^{l+1}-1 \rightarrow 5 \cdot 2^{l+1}-3\right)$ is trivial $\bmod F^{4}$ and in turn $W_{l}(H)=\{1\}$. Thus by Lemma $5, \delta_{3 l+4}(H)=\{1\}$. Now by Lemma $2(\mathrm{ii}), \delta_{3 l+6}\left(F_{\infty}\left(\mathfrak{B}_{4}\right)\right)=\{1\}$, as was required.

\section{REFERENCES}

1. Narain D. Gupta and Seán J. Tobin, On certain groups with exponent four, Math. Z. 102 (1967), 216-226. MR 36 \#5220.

2. Narain D. Gupta and Kenneth W. Weston, On groups of exponent four, J. Algebra 17 (1971), 59-66.

3. C. R. B. Wright, On the nilpotency class of a group of exponent four, Pacific J. Math. 11 (1961), 387-394. MR 23 \#A927.

Department of Mathematics, University of Manitoba, Winnipeg, Manitoba, Canada 